\title{
Killing Vector Fields, Maxwell Equations and Lorentzian Spacetimes*
}

\author{
Waldyr A. Rodrigues Jr. \\ Institute of Mathematics, Statistics and Scientific Computation \\ IMECC-UNICAMP CP 6065 \\ 13083-859 Campinas, SP, Brazil \\ e-mail: walrod@ime.unicamp.br
}

November 4, 2018

\begin{abstract}
In this paper we first analyze the structure of Maxwell equations in a Lorentzian spacetime when the potential is $A=\mathfrak{e} K$, i.e., proportional to a 1 -form $K$ physically equivalent Killing vector field. We show that $A$ necessarily obeys the Lorenz gauge $\delta A=0$. Moreover we determine the form of the current associated with this potential showing that it is of a superconducting type, i.e., proportional to the potential and given by $2 \mathcal{R}_{\beta} A^{\beta}$, where the $\mathcal{R}_{\beta}$ are the Ricci 1-form fields. Finally we study the structure of the spacetime generated by the coupled system consisting of a electromagnetic field $F=d A$ (with $A=\mathfrak{e} K$ ), an ideal charged fluid with dynamics described by an action function $S$ and the gravitational field. We show that Einstein equations is then equivalent to Maxwell equations with a current given by $f F A F$ (the product meaning the Clifford product of the corresponding fields), where $f$ is a scalar function which satisfies a well determined algebraic quadratic equation.
\end{abstract}

\section{Introduction}

In a previous paper [7] we study using the Clifford bundle formalism the effective Lorentzian and teleparallel spacetimes generated by a electromagnetic field moving in Minkowski spacetime.

Here, using the same mathematical apparatus, we study another intriguing connections between gravitation described by Einstein field equations and electromagnetism described by Maxwell equations. In order to do that we first prove in Section 2 a proposition showing that if $\mathbf{K}$ is a Killing vector field on a Lorentzian manifold $(M, \mathbf{g})$ then the form field $K=\mathbf{g}(\mathbf{K}$,$) satisfies \delta K=0$ and

* Paper presented at the $8^{\text {th }}$ International Conference on Clifford Algebras and their Applications in Mathematical Physics (ICCA8), Campinas, May 26-30 2008. 
a wave equation given by Eq.(2) in terms of the covariant D' Alembertian. We also show that the Ricci operator (which can be defined only in the Clifford bundle of differential forms) applied to $K$ it is equal to the covariant D'Alembertian applied to $K$. Next, in Section 3 we analyze the structure of Maxwell equations in a Lorentzian spacetime when the potential obeys the Lorenz gauge. Take notice that if a potential is in Lorenz gauge this does not necessarily implies that it is a 1-form physically equivalent to a Killing vector field. Moreover we determine the form of the current associated with this potential $A$ showing that it is given by $2 A^{\beta} \mathcal{R}_{\beta}$, where the $\mathcal{R}_{\beta}$ are the Ricci 1-form fields (Eq.(64)) In Section 4 we study the structure of the Lorentzian spacetime representing the gravitational field produced and interacting with an electromagnetic field $F=d A$ (where $A$ is proportional $K=\boldsymbol{g}(\mathbf{K}$, ), with $\mathbf{K}$ a Killing vector field) generated by an ideal charged current $J_{e}$. We show that Einstein equations is in this case represented by Maxwell equations with a current given by $f F A F$ (the product being intended as the Clifford product of the corresponding fields), where $f$ is a scalar function solution of a well determined algebraic quadratic equation (Eq. (36). In Section 5 we present our conclusions and in the Appendix we recall the main definitions and formulas of the Clifford bundle formalism, proving a result that is need in the proof of Proposition 1.

\section{Some Preliminaries}

In this paper a spacetime structure is a pentuple $\mathfrak{M}=\left(M, \mathbf{g}, D, \tau_{g}, \uparrow\right)$ where $\left(M, \boldsymbol{g}, \tau_{g}\right)$ is a Lorentzian manifold, $D$ is the Levi-Civita connection of $\boldsymbol{g}$ and $\uparrow$ is an equivalence relation between timelike vector fields defining the time orientation 2 . Also, $\mathrm{g} \in \sec T_{2}^{0} M$ denotes the metric of the cotangent bundle, $\wedge T^{*} M$ denotes the bundle of (nonhomogeneous) differential forms and $\mathcal{C} \ell(M, \mathrm{~g})$ denotes the Clifford bundle of differential forms. We shall take advantage of the well known fact that [5] $\wedge T^{*} M \hookrightarrow \mathcal{C} \ell(M, \mathrm{~g})$ and use in our calculations the powerful Clifford bundle formalism [9]. Let $\left\{\partial_{\mu}\right\}, \partial_{\mu}:=\frac{\partial}{\partial x^{\mu}}$ be an arbitrary coordinate basis for $T U \subset T M$ and $\left\{\gamma^{\mu}=d x^{\mu}\right\}$ the corresponding dual basis of $T^{*} U \subset T^{*} M=\bigwedge^{1} T^{*} M$. As explained in the Appendix the $\gamma^{\mu}$ will be though as sections of the Clifford bundle, more precisely, $\gamma^{\mu} \in \sec T^{*} U \subset$ $\sec \bigwedge^{1} T^{*} M \hookrightarrow \mathcal{C} \ell(M, \mathrm{~g})$. Also, we recall that the set $\left\{\partial^{\mu}\right\}, \partial^{\mu}=g^{\mu \nu} \frac{\partial}{\partial x^{\mu}} \in$ $\sec T M$ such that $\boldsymbol{g}\left(\partial_{\mu}, \partial^{\nu}\right)=\delta_{\nu}^{\mu}$ is called the reciprocal basis of $\left\{\partial_{\mu}\right\}$ and the set $\left\{\gamma_{\mu}\right\}$ such that $\mathrm{g}\left(\gamma^{\mu}, \gamma_{\nu}\right)=\delta_{\nu}^{\mu}$ is called the reciprocal basis of $\left\{\gamma^{\mu}\right\}$. We denote $\mathrm{g}\left(\gamma^{\mu}, \gamma_{\nu}\right)=\gamma^{\mu} \cdot \gamma_{\nu}$, where $\cdot$ denotes the scalar product in $\mathcal{C} \ell(M, \mathrm{~g})$. Finally, $\boldsymbol{\partial}=\gamma^{\mu} D_{\partial_{\mu}}$ denotes the Dirac operator acting on sections of $\mathcal{C} \ell(M, \mathrm{~g})$ and $\square=\boldsymbol{\partial} \cdot \boldsymbol{\partial}$ and $\boldsymbol{\partial} \wedge \boldsymbol{\partial}$ denotes respectively the covariant D'Alembertian and the Ricci operators. The operator $\diamond=\partial^{2}$ is called Hodge D'Alembertian and

\footnotetext{
${ }^{1}$ Our result differs from a factor of 2 from the one presented in 8 and also in [?], where an electromagnetic potential proportional to a Killing vector field is called a Papapetrou field. The important discrepancy is due to the fact that those authors identified the electromagnetic current $J_{e}$ with $\square A$ instead of identifying it with $-\delta d A$, as it must be. See the text for details.

${ }^{2}$ Details may be found, e.g., in [9] 11 ]
} 
the relations between those operators and their main properties are presented in the Appendix.

Proposition 1 Let $\mathbf{K} \in \sec T M$ be a Killing vector field, i.e., $£_{\mathbf{K}} \mathbf{g}=0$. Let $K=\mathbf{g}(\mathbf{K}$,$) . Then$

$$
\begin{aligned}
\delta K & =0, \\
\partial \wedge \partial K & =K_{\alpha} \mathcal{R}^{\alpha}, \\
\square K & =K_{\alpha} \mathcal{R}^{\alpha},
\end{aligned}
$$

where $\mathcal{R}^{\alpha} \in \sec \bigwedge^{1} T^{*} M \hookrightarrow \mathcal{C} \ell(M, \mathrm{~g})$ are the Ricci 1-forms given by Eq.(64).

Proof To prove Eq.(1) it is only necessary to recall that since

$$
£_{\mathbf{K}} \mathbf{g}=0 \Leftrightarrow D_{\mu} K_{\nu}+D_{\nu} K_{\mu}=0
$$

and by Eq.(58) $\delta K=-\boldsymbol{\partial}\lrcorner K$ we have

$$
\begin{aligned}
\delta K & \left.=-\gamma^{\mu}\right\lrcorner D_{e_{\mu}} K \\
& \left.=-\gamma^{\mu}\right\lrcorner\left[\left(D_{\mu} K_{\nu}\right) \gamma^{\nu}\right] \\
& =g^{\mu \nu} D_{\mu} K_{\nu}=\frac{1}{2} g^{\mu \nu}\left(D_{\mu} K_{\nu}+D_{\nu} K_{\mu}\right)=0 .
\end{aligned}
$$

The proof of Eq.(2) is trivial. Indeed, the Ricci operator is extensorial [9], i.e., according to Eq. (67) satisfies

$$
\partial \wedge \partial K=K_{\mu} \partial \wedge \partial \gamma^{\mu}
$$

and thus using Eq.(64) we get:

$$
\partial \wedge \partial K=\mathcal{R}_{\mu} K^{\mu} .
$$

To prove Eq.(3) we use Eq.(62) and write

$$
\boldsymbol{\partial} \cdot \boldsymbol{\partial} K=g^{\sigma \nu} D_{\sigma} D_{\nu} K_{\mu} \gamma^{\mu}
$$

Now, we calculate $D_{\sigma} D_{\nu} K_{\alpha}$. Since $\mathbf{K}$ is a Killing vector fied satisfying Eq.(44) we can write

$$
\begin{aligned}
& D_{\sigma}\left(D_{\nu} K_{\mu}+D_{\mu} K_{\nu}\right) \\
& =\left[D_{\sigma}, D_{\nu}\right] K_{\mu}+D_{\nu} D_{\sigma} K_{\mu}+\left[D_{\sigma}, D_{\mu}\right] K_{\nu}+D_{\mu} D_{\sigma} K_{\nu}=0 .
\end{aligned}
$$

Taking into account that

$$
\begin{aligned}
g^{\sigma \nu}\left[D_{\sigma}, D_{\nu}\right] K_{\mu} & =0, \\
g^{\sigma \nu} D_{\mu} D_{\sigma} K_{\nu} & =\frac{1}{2} g^{\sigma \nu} D_{\mu}\left(D_{\sigma} K_{\nu}+D_{\nu} K_{\sigma}\right)=0, \\
g^{\sigma \nu}\left[D_{\sigma}, D_{\mu}\right] K_{\nu} & =-g^{\sigma \nu} R_{\nu}{ }^{\rho}{ }_{\sigma \mu} K_{\rho}=-g^{\sigma \nu} R_{\nu \rho \sigma \mu} K^{\rho} \\
& =-g^{\sigma \nu} R_{\rho \nu \mu \sigma} K^{\rho}=-R_{\rho \mu} K^{\rho},
\end{aligned}
$$


we get on multiplying Eq.(7) by $g^{\sigma \nu}$ that

$$
g^{\sigma \nu} D_{\nu} D_{\sigma} K_{\mu}=R_{\rho \mu} K^{\rho}
$$

and thus

$$
\partial \cdot \partial K=g^{\sigma \nu} D_{\sigma} D_{\nu} K_{\mu} \gamma^{\mu}=R_{\rho \mu} K^{\rho} \gamma^{\mu}=K^{\rho} \mathcal{R}_{\alpha} .
$$

Corollary 2 Call $M=d K$. Then

$$
J=:-\delta M=2 K^{\beta} \mathcal{R}_{\beta}
$$

Proof Indeed, we have recalling Eq.(11) and Eq.(60) that

$$
\begin{aligned}
-\delta d K & =-\delta d K-d \delta K=(d-\delta)(d-\delta) K=\boldsymbol{\partial}^{2} K \\
& =\boldsymbol{\partial} \cdot \boldsymbol{\partial} K+\boldsymbol{\partial} \wedge \boldsymbol{\partial} K \\
& =K^{\beta} \mathcal{R}_{\beta}+K^{\beta} \mathcal{R}_{\beta}=2 K^{\beta} \mathcal{R}_{\beta},
\end{aligned}
$$

and the result is proved.

\section{$3 \quad$ Electrodynamics on $\mathfrak{M}$}

As it is well known in General Relativity (GR) the gravitational field generated by an energy momentum tensor $\mathbf{T}=T_{\mu} \otimes \gamma^{\mu}$, (where the $T_{\mu}=T_{\mu}^{\nu} \boldsymbol{\gamma}_{\nu} \in$ sec $\bigwedge^{1} T^{*} M \hookrightarrow \mathcal{C} \ell(M, \mathrm{~g})$ are the energy-momentum1-form fields) is represented by a Lorentzian spacetime $\mathfrak{M}=\left(M, \mathbf{g}, D, \tau_{g}, \uparrow\right)$. Let $\mathfrak{e}$ be a constant with the physical dimension of an electromagnetic potential and let

$$
A=\mathfrak{e} K
$$

be an electromagnetic potential.

Suppose now that a probe electromagnetic field $F=d A \in \sec \bigwedge^{2} T^{*} M \hookrightarrow$ $\mathcal{C} \ell(M, \mathrm{~g})$ generated by a (probe) current $J_{e} \in \sec \bigwedge^{1} T^{*} M \hookrightarrow \mathcal{C} \ell(M, \mathrm{~g})$, lives and develops its dynamics in $\mathfrak{M}$. Then we assume as usual that $F$ satisfies Maxwell equations

$$
d F=0, \delta F=-J_{e}
$$

which taking into account the definition of the Dirac operator (Eq. (53) ) can be written as a single equation

$$
\partial F=J_{e}
$$

Now, it is usual in electrodynamics problems to work with the potential $A$ and fix the Lorenz gauge $\delta A=0$. This is done, e.g., in the classical Eddington book 2]. Taking into account Eq.(1) of Proposition 1 we thus have that in a spacetime $\mathfrak{M}=\left(M, \mathbf{g}, D, \tau_{g}, \uparrow\right)$ a probe electromagnetic field $F=d A$ such that $\mathbf{A}=\mathrm{g}(A$, ) is a Killing vector field is such that $\delta A=0$, i.e., it is in the Lorentz gauge. Moreover. using Eq.(9) we see that the current $J_{e}=2 \mathcal{R}_{\beta} A^{\beta}$ is of the superconductor type, i.e., proportional to the potential $A$. At the spacetime points where the Ricci tensor is zero we necessarily have a null current. 
This result is important since permit us to determine for each Killing vector in $\mathfrak{M}$ a special current of superconductor type.

These results shows that in GR the potential $A$ appears to acquire a status that it does not have in Special Relativity. In the reamining of the paper we study further consequences of supposing that $A$ is proportional to a Killing vector field

Remark 3 We recall that if $L \in \sec \bigwedge^{1} T^{*} M \hookrightarrow \mathcal{C} \ell(M, \mathrm{~g})$ is such that $\delta L=0$ we have that

$$
\frac{1}{2} g^{\kappa \mu}\left(D_{\kappa} L_{\mu}+D_{\mu} L_{k}\right)=0
$$

which however does not implies in general that $D_{\kappa} L_{\mu}+D_{\mu} L_{k}=0$, i.e., that $\mathbf{L}=\mathrm{g}(L$,$) is a Killing vector field.$

\section{The Spacetime $\mathfrak{M}$ Generated by an Ideal Cur- rent Interacting with the Electromagnetic Field and the Gravitational Field}

In this section we analyze the dynamics of a coupled system consisting of an ideal charged matter field plus electromagnetic field and the gravitational field. For simplicity we restrict ourselves to the case of an incompressible charged and frictionless fluid represented by a velocity field $\mathbf{V} \in \sec T M$ with $\mathbf{g}(\mathbf{V}, \mathbf{V})=1$ and such that each one of its integral lines, say $\sigma: \tau \mapsto \sigma(\tau) \in M$ is such that $\left.\mathbf{V}\right|_{\sigma}=\sigma_{*}=d / d \tau$ obeys the Lorentz force equation, which writing $v=\boldsymbol{g}\left(\sigma_{*},\right)$ reads:

$$
\left.D_{\sigma_{\star}} v=\frac{e}{m} v\right\lrcorner F
$$

where $e$ and $m$ are the charge and mass of the charged particles composing the ideal charged fluid and $F$ is the total electromagnetic field generated by it.

Introducing the velocity 1 -form field $V=\mathbf{g}(\mathbf{V}$,$) and using the noticeable$ identity given by Eq.(59) we can write the first member of Eq.(15) as

$$
\begin{aligned}
D_{\sigma_{\star}} v & \left.=v^{\mu} D_{\partial_{\mu}} v=(V\lrcorner \boldsymbol{\partial}\right)\left.V\right|_{\sigma} \\
& =V\lrcorner\left.(\boldsymbol{\partial} \wedge V)\right|_{\sigma}
\end{aligned}
$$

and thus since $F=d A$ and $\partial \wedge V=d V$, Eq.(15) implies that the velocity field satisfies the equation

$$
V\lrcorner[d(m V-e A)]=0 .
$$

A sufficient condition for the validity of Eq.(18) is the existence of a 0 -form field $S$ such that

$$
m V-e A=d S
$$

Then,

$$
d S+e A=m V
$$


and

$$
(d S+e A)^{2}=m^{2}
$$

which we recognize as the classical Hamilton-Jacobi equation. Before proceeding we recall that since $\delta V=0$ for a perfect incompressible charged fluid, we get form Eq.(19)

$$
\delta d S+d \delta S+e \delta A=0,
$$

i.e.,

$$
\partial^{2} S=e \delta A
$$

Remark 4 Eq.(22) implies that the charged particle fluid action $S$ when the potential $A$ is in the Lorenz gauge satisfies a homogeneous wave equation, even if $A$ is not proportional to a Killing vector field.

Then in what follows we call $S$ the action of the ideal charged fluid. Moreover, since we are here more interested in the structure of the field equations, we choose the mass and the charge of the fluid particles to be in our system of units $m=1$ and $e=1$. Also the gravitational constant is 1 in our units.

Now, the Lagrangian density for the charged fluid model interacting with the electromagnetic field and the gravitational field is given by

$$
\mathcal{L}=-\frac{1}{2} J_{e} \wedge \star J_{e}-\frac{1}{2} F \wedge \star F+\mathcal{L}_{E H},
$$

where

$$
\mathcal{L}_{E H}=\frac{1}{2}\left[\mathcal{R}_{\mu \nu} \wedge \star\left(\gamma^{\mu} \wedge \gamma^{\nu}\right)\right]
$$

is the Einstein-Hilbert Lagrangian density and

$$
J_{e}=(d S+A) .
$$

The equations of motion resulting from the principle of stationary action are 12]:

$$
\begin{aligned}
\delta J_{e} & =0, \\
\delta F & =-J_{e}, \\
\star \mathcal{G}_{\alpha} & =-\star T_{\alpha}-\star t_{\alpha} .
\end{aligned}
$$

where

$$
\star T_{\beta}=-\frac{1}{2} \star\left(F \gamma_{\beta} F\right)
$$

are the energy-momentum 3-form fields of the electromagnetic field (see, e.g., [9, 7. ) and the

$$
\begin{aligned}
\star t_{\alpha} & =\frac{1}{2}\left[\left(\boldsymbol{\gamma}_{\alpha} \cdot J_{e}\right) \wedge \star J_{e}+J_{e} \wedge\left(\boldsymbol{\gamma}_{\alpha} \cdot \star J_{e}\right)\right. \\
& =\frac{1}{2} \star\left(J_{e} \gamma_{\alpha} J_{e}\right)
\end{aligned}
$$


are the energy-momentum 3-form fields of the ideal charged fluid. Note that Eq. (26) reproduces trivially Eq.(19).

Now, it is easy to verify that Einstein equations implies that

$$
R=J_{e}^{2} .
$$

Then, we can rewrite Eq.(10) as

$$
\begin{aligned}
d \star d A & =-2 \star A^{\beta}\left(\mathcal{R}_{\beta}-\frac{1}{2} R \gamma_{\beta}\right)-A^{\beta} R \star \gamma_{\beta} \\
& =-\star 2 A^{\beta} \mathcal{G}_{\beta}+J_{e}^{2} \star A \\
& =2 \star\left[-\frac{1}{2} F A F+\frac{1}{2} J_{e} A J_{e}\right)+J_{e}^{2} \star A \\
& =\star-(F A F)-\star A J_{e}^{2}+2\left(A \cdot J_{e}\right) \star J_{e}+J_{e}^{2} \star A \\
& =\star-(F A F)+2\left(A \cdot J_{e}\right) \star J_{e}
\end{aligned}
$$

from where, since

$$
\delta F=-J_{e}, d F=0
$$

we see that the current $J_{e}$ must satisfy the following equation

$$
J_{e}=-F A \tilde{F}+2\left(A \cdot J_{e}\right) J_{e} .
$$

If we make the exterior multiplication of both members of Eq. (34) by $J_{e}$ we get that $(F A \tilde{F}) \wedge J_{e}=0$, from where taking into account that $B=F A \tilde{F} \in$ $\sec \bigwedge^{1} T^{\star} M$ implies that

$$
J_{e}=f F A F=-f B,
$$

where $f$ is a scalar function that in order for Eq. (34) to be satisfied must solve, when $B^{2} \neq 0$, the quadratic equation

$$
2(A \cdot B) f^{2}+f+1=0,
$$

which has real roots only if $(A \cdot B)^{2} \leq \frac{1}{8}$. We then have the

Proposition 5 The Lorentzian spacetime $\mathfrak{M}=\left(M, \mathbf{g}, D, \tau_{g}, \uparrow\right)$ where an incompressible charged fluid described by an action $S$ generates an electromagnetic field $F=d A$ such that $\mathbf{A}=\mathrm{g}(A$,$) is proportional to a Killing vector field and$ both are in interaction with the gravitational field is such that and the current is given by $J_{e}=f F A F$ where $f$ is a solution of the algebraic quadratic equation given by Eq.(36) with $[A \cdot(F A F)]^{2} \leq \frac{1}{8}$. Moreover, Einstein equations are equivalent to Maxwell equations given by Eq.(32). 


\section{Conclusions}

In this paper using the Clifford bundle formalism and a proposition (Proposition 1) of differential geometry which shows that if $\mathbf{K}$ is a Killing vector field on a Lorentzian manifold $(M, \mathbf{g})$ then the form field $K=\mathbf{g}(\mathbf{K}$,$) satisfies \delta K=0$ and a wave equation given by Eq.(2) in terms of the covariant D' Alembertian applied to $K$. We also showed that the Ricci operator, which can be defined only in the Clifford bundle of differential forms applied to $K$ is equal to the covariant D'Alembertian applied to $K$. We analyzed morevoer the structure of Maxwell equations in a Lorentzian spacetime when the potential is proportional to $K, A=\mathfrak{e} K$ and thus satisfies the Lorenz gauge $\delta A=0$. The explicit form of the current which generates the electromagnetic field has been calculated and resulted proportional to $F A F$. Next we studied the structure of the spacetime generated by the interaction of the a perfect charged fluid described by action $S$, its electromagnetic field $F=d A$, with $A=\mathfrak{e} K$ and the gravitational field. We found that Einstein equations for this case is represented by Maxwell equations with a current given by Proposition 4 , i.e., $J_{e}=f F A F$, where $f$ is a scalar function which is solution of a well determined quadratic equation.

\section{A Clifford Bundle Formalism}

Let $\mathfrak{M}=\left(M, \mathfrak{g}, D, \tau_{g}, \uparrow\right)$ be an arbitrary Lorentzian spacetime. The quadruple $\left(M, \mathbf{g}, \tau_{g}, \uparrow\right)$ denotes a four-dimensional time-oriented and space-oriented Lorentzian manifold [9, 11. This means that $g \in \sec T_{2}^{0} M$ is a Lorentzian metric of signature $(1,3), \tau_{g} \in \sec \bigwedge^{4} T^{*} M$ and $\uparrow$ is a time-orientation (see details, e.g., in [11). Here, $T^{*} M[T M]$ is the cotangent [tangent] bundle. $T^{*} M=\cup_{x \in M} T_{x}^{*} M, T M=\cup_{x \in M} T_{x} M$, and $T_{x} M \simeq T_{x}^{*} M \simeq \mathbb{R}^{1,3}$, where $\mathbb{R}^{1,3}$ is the Minkowski vector space 3 is the Levi-Civita connection of $\boldsymbol{g}$, i.e., it is metric compatible connection, i.e., $D \boldsymbol{g}=0$, and in general, $\mathbf{R}=\mathbf{R}^{D} \neq 0$, and $\Theta=\Theta^{D}=0, \mathbf{R}$ and $\Theta$ being respectively the curvature and torsion tensors of the connection. Minkowski spacetime is the particular case of a Lorentzian spacetime for which $\mathbf{R}=0, \Theta=0$, and $M \simeq \mathbb{R}^{4}$. Let $\mathrm{g} \in \sec T_{0}^{2} M$ be the metric of the cotangent bundle. The Clifford bundle of differential forms $\mathcal{C}(M, \mathrm{~g})$ is the bundle of algebras, i.e., $\mathcal{C} \ell(M, \mathrm{~g})=\cup_{x \in M} \mathcal{C}\left(T_{x}^{*} M, \mathrm{~g}\right)$, where $\forall x \in M, \mathcal{C l}\left(T_{x}^{*} M, \mathrm{~g}\right)=\mathbb{R}_{1,3}$, the so called spacetime algebra [9]. Recall also that $\mathcal{C} \ell(M, \mathrm{~g})$ is a vector bundle associated to the orthonormal frame bundle, i.e., $\mathcal{C} \ell(M, \mathrm{~g})=P_{\mathrm{SO}_{(1,3)}^{e}}(M) \times_{\mathrm{Ad}} \mathcal{C} l_{1,3}$ [5, 6]. For any $x \in M, \mathcal{C} \ell\left(T_{x}^{*} M,\left.\mathrm{~g}\right|_{x}\right)$ as a linear space over the real field $\mathbb{R}$ is isomorphic to the Cartan algebra $\wedge T_{x}^{*} M$ of the cotangent space. $\bigwedge T_{x}^{*} M=\oplus_{k=0}^{4} \bigwedge^{k} T_{x}^{*} M$, where $\bigwedge^{k} T_{x}^{*} M$ is the $\left(\begin{array}{l}4 \\ k\end{array}\right)$ dimensional space of $k$-forms. Then, sections of $\mathcal{C} \ell(M, \mathrm{~g})$ can be represented as a sum of non homogeneous differential forms, that will be called Clifford (multiform) fields. In the Clifford bundle formalism, of course, arbitrary basis can be used, but in this short review of the main ideas of the Clifford calculus we use

\footnotetext{
${ }^{3}$ Not to be confused with Minkowski spacetime [11].
} 
orthonormal basis. Let then $\left\{\mathbf{e}_{\mathbf{a}}\right\}$ be an orthonormal basis for $T U \subset T M$, i.e., $\mathrm{g}\left(\mathbf{e}_{\mathbf{a}}, \mathbf{e}_{\mathbf{a}}\right)=\eta_{\mathbf{a b}}=\operatorname{diag}(1,-1,-1,-1)$. Let $\boldsymbol{\theta}^{\mathbf{a}} \in \sec \bigwedge^{1} T^{*} M \hookrightarrow \sec \mathcal{C} \ell(M, \mathrm{~g})$ $(\mathbf{a}=0,1,2,3)$ be such that the set $\left\{\boldsymbol{\theta}^{\mathbf{a}}\right\}$ is the dual basis of $\left\{\mathbf{e}_{\mathbf{a}}\right\}$.

\section{A.1 Clifford Product}

The fundamental Clifford product (in what follows to be denoted by juxtaposition of symbols) is generated by

$$
\boldsymbol{\theta}^{\mathbf{a}} \boldsymbol{\theta}^{\mathbf{b}}+\boldsymbol{\theta}^{\mathbf{b}} \boldsymbol{\theta}^{\mathbf{a}}=2 \eta^{\mathbf{a b}}
$$

and if $\mathcal{C} \in \sec \mathcal{C} \ell(M, \mathrm{~g})$ we have

$$
\mathcal{C}=s+v_{\mathbf{a}} \boldsymbol{\theta}^{\mathbf{a}}+\frac{1}{2 !} f_{\mathbf{a b}} \boldsymbol{\theta}^{\mathbf{a}} \boldsymbol{\theta}^{\mathbf{b}}+\frac{1}{3 !} t_{\mathbf{a b c}} \boldsymbol{\theta}^{\mathbf{a}} \boldsymbol{\theta}^{\mathbf{b}} \boldsymbol{\theta}^{\mathbf{c}}+p \boldsymbol{\theta}^{5},
$$

where $\tau_{g}=\boldsymbol{\theta}^{5}=\boldsymbol{\theta}^{0} \boldsymbol{\theta}^{\mathbf{1}} \boldsymbol{\theta}^{\mathbf{2}} \boldsymbol{\theta}^{\mathbf{3}}$ is the volume element and $s, v_{\mathbf{a}}, f_{\mathbf{a b}}, t_{\mathbf{a b c}}, p \in$ $\sec \bigwedge^{0} T^{*} M \hookrightarrow \sec \mathcal{C l}(M, \mathrm{~g})$.

For $A_{r} \in \sec \bigwedge^{r} T^{*} M \hookrightarrow \sec \mathcal{C l}(M, \mathrm{~g}), B_{s} \in \sec \bigwedge^{s} T^{*} M \hookrightarrow \sec \mathcal{C}(M, \mathrm{~g})$ we define the exterior product in $\mathcal{C}(M, \mathrm{~g}) \quad(\forall r, s=0,1,2,3)$ by

$$
A_{r} \wedge B_{s}=\left\langle A_{r} B_{s}\right\rangle_{r+s}
$$

where \langle\rangle$_{k}$ is the component in $\wedge^{k} T^{*} M$ of the Clifford field. Of course, $A_{r} \wedge B_{s}=(-1)^{r s} B_{s} \wedge A_{r}$, and the exterior product is extended by linearity to all sections of $\mathcal{C}(M, \mathrm{~g})$.

Let $A_{r} \in \sec \bigwedge^{r} T^{*} M \hookrightarrow \sec \mathcal{C l}(M, \mathrm{~g}), B_{s} \in \sec \bigwedge^{s} T^{*} M \hookrightarrow \sec \mathcal{C l}(M, \mathrm{~g})$. We define a scalar product in $\mathrm{Cl}(M, \mathrm{~g})$ (denoted by $\cdot$ ) as follows:

(i) For $a, b \in \sec \bigwedge^{1} T^{*} M \hookrightarrow \sec \mathcal{C}(M, \mathrm{~g})$,

$$
a \cdot b=\frac{1}{2}(a b+b a)=\mathrm{g}(a, b) .
$$

(ii) For $A_{r}=a_{1} \wedge \ldots \wedge a_{r}, B_{r}=b_{1} \wedge \ldots \wedge b_{r}, a_{i}, b_{j} \in \sec \wedge^{1} T^{*} M \hookrightarrow \sec \mathcal{C}(M, \mathrm{~g})$, $i, j=1, \ldots, r$,

$$
\begin{aligned}
A_{r} \cdot B_{r} & =\left(a_{1} \wedge \ldots \wedge a_{r}\right) \cdot\left(b_{1} \wedge \ldots \wedge b_{r}\right) \\
& =\left|\begin{array}{lll}
a_{1} \cdot b_{1} & \ldots . & a_{1} \cdot b_{r} \\
\ldots \ldots \ldots \ldots & \ldots . & \ldots \ldots \ldots \ldots \\
a_{r} \cdot b_{1} & \ldots . & a_{r} \cdot b_{r}
\end{array}\right| .
\end{aligned}
$$

We agree that if $r=s=0$, the scalar product is simply the ordinary product in the real field.

Also, if $r \neq s$, then $A_{r} \cdot B_{s}=0$. Finally, the scalar product is extended by linearity for all sections of $\mathcal{C}(M, \mathrm{~g})$.

For $r \leq s, A_{r}=a_{1} \wedge \ldots \wedge a_{r}, B_{s}=b_{1} \wedge \ldots \wedge b_{s}$, we define the left contraction \lrcorner$\left.:\left(A_{r}, B_{s}\right) \mapsto A_{r}\right\lrcorner B_{s}$ by

$$
\left.A_{r}\right\lrcorner B_{s}=\sum_{i_{1}<\ldots<i_{r}} \epsilon^{i_{1} \ldots i_{s}}\left(a_{1} \wedge \ldots \wedge a_{r}\right) \cdot\left(b_{i_{1}} \wedge \ldots \wedge b_{i_{r}}\right) \sim b_{i_{r}+1} \wedge \ldots \wedge b_{i_{s}}
$$


where $\sim$ is the reverse mapping (reversion) defined by $\sim \sec \mathcal{C}(M, \mathrm{~g}) \rightarrow$ $\sec \mathcal{C}(M, \mathrm{~g})$. For any $X=\bigoplus_{p=0}^{4} X_{p}, X_{p} \in \sec \bigwedge^{p} T^{*} M \hookrightarrow \sec \mathcal{C}(M, \mathrm{~g})$,

$$
\tilde{X}=\sum_{p=0}^{4} \tilde{X}_{p}=\sum_{p=0}^{4}(-1)^{\frac{1}{2} k(k-1)} X_{p} .
$$

We agree that for $\alpha, \beta \in \sec \bigwedge^{0} T^{*} M$ the contraction is the ordinary (pointwise) product in the real field and that if $\alpha \in \sec \bigwedge^{0} T^{*} M, X_{r} \in \sec \bigwedge^{r} T^{*} M, Y_{s} \in$ $\sec \bigwedge^{s} T^{*} M \hookrightarrow \sec \mathcal{C}(M, \mathrm{~g})$ then $\left.\left.\left(\alpha X_{r}\right)\right\lrcorner B_{s}=X_{r}\right\lrcorner\left(\alpha Y_{s}\right)$. Left contraction is extended by linearity to all pairs of sections of $\mathcal{C}(M, \mathrm{~g})$, i.e., for $X, Y \in \sec \mathcal{C}(M, \mathrm{~g})$

$$
\left.X\lrcorner Y=\sum_{r, s}\langle X\rangle_{r}\right\lrcorner\langle Y\rangle_{s}, \quad r \leq s .
$$

It is also necessary to introduce the operator of right contraction denoted by $\llcorner$. The definition is obtained from the one presenting the left contraction with the imposition that $r \geq s$ and taking into account that now if $A_{r} \in \sec \bigwedge^{r} T^{*} M$, $B_{s} \in \sec \bigwedge^{s} T^{*} M$ then $A_{r}\left\llcorner\left(\alpha B_{s}\right)=\left(\alpha A_{r}\right)\left\llcorner B_{s}\right.\right.$. See also the third formula in Eq.(45).

The main formulas used in this paper can be obtained from the following ones

$$
\begin{aligned}
a \mathcal{B}_{s} & =a\lrcorner \mathcal{B}_{s}+a \wedge \mathcal{B}_{s}, \quad \mathcal{B}_{s} a=\mathcal{B}_{s}\left\llcorner a+\mathcal{B}_{s} \wedge a,\right. \\
a\lrcorner \mathcal{B}_{s} & =\frac{1}{2}\left(a \mathcal{B}_{s}-(-1)^{s} \mathcal{B}_{s} a\right), \\
\left.\mathcal{A}_{r}\right\lrcorner \mathcal{B}_{s} & =(-1)^{r(s-r)} \mathcal{B}_{s}\left\llcorner\mathcal{A}_{r},\right. \\
a \wedge \mathcal{B}_{s} & =\frac{1}{2}\left(a \mathcal{B}_{s}+(-1)^{s} \mathcal{B}_{s} a\right), \\
\mathcal{A}_{r} \mathcal{B}_{s} & =\left\langle\mathcal{A}_{r} \mathcal{B}_{s}\right\rangle_{|r-s|}+\left\langle\mathcal{A}_{r} \mathcal{B}_{s}\right\rangle_{|r-s|+2}+\ldots+\left\langle\mathcal{A}_{r} \mathcal{B}_{s}\right\rangle_{|r+s|} \\
& =\sum_{k=0}^{m}\left\langle\mathcal{A}_{r} \mathcal{B}_{s}\right\rangle_{|r-s|+2 k} \\
\mathcal{A}_{r} \cdot \mathcal{B}_{r} & \left.=\mathcal{B}_{r} \cdot \mathcal{A}_{r}=\widetilde{\mathcal{A}}_{r}\right\lrcorner \mathcal{B}_{r}=\mathcal{A}_{r}\left\llcorner\widetilde{\mathcal{B}}_{r}=\left\langle\widetilde{\mathcal{A}}_{r} \mathcal{B}_{r}\right\rangle_{0}=\left\langle\mathcal{A}_{r} \widetilde{\mathcal{B}}_{r}\right\rangle_{0} .\right.
\end{aligned}
$$

Two other important identities used in the main text are:

$$
\begin{aligned}
a\lrcorner(\mathcal{X} \wedge \mathcal{Y}) & =(a\lrcorner \mathcal{X}) \wedge \mathcal{Y}+\hat{\mathcal{X}} \wedge(a\lrcorner \mathcal{Y}), \\
A\lrcorner(B\lrcorner C) & =(A \wedge B)\lrcorner C,
\end{aligned}
$$

for any $a \in \sec \bigwedge^{1} T^{*} M \hookrightarrow \mathcal{C} \ell(M, \mathrm{~g})$ and $\mathcal{X}, \mathcal{Y} \in \sec \bigwedge T^{*} M \hookrightarrow \mathcal{C} \ell(M, \mathrm{~g})$, and for any $A, B, C \in \sec \wedge T^{*} M \hookrightarrow \mathcal{C} \ell(M, \mathrm{~g})$. 


\section{A.1.1 Hodge Star Operator}

Let $\star$ be the Hodge star operator, i.e., the mapping $\star: \bigwedge^{k} T^{*} M \rightarrow \bigwedge^{4-k} T^{*} M$, $A_{k} \mapsto \star A_{k}$. For $A_{k} \in \sec \bigwedge^{k} T^{*} M \hookrightarrow \sec \mathcal{C}(M, \mathrm{~g})$ we have

$$
\left[B_{k} \cdot A_{k}\right] \tau_{\mathrm{g}}=B_{k} \wedge \star A_{k}, \forall B_{k} \in \sec \bigwedge^{k} T^{*} M \hookrightarrow \sec \mathcal{C}(M, \mathrm{~g}) .
$$

where $\tau_{\mathrm{g}}=\theta^{5} \in \sec \bigwedge^{4} T^{*} M \hookrightarrow \sec \mathcal{C}(M, \mathrm{~g})$ is a standard volume element. We have,

$$
\left.\star A_{k}=\widetilde{A}_{k} \tau_{\mathrm{g}}=\widetilde{A}_{k}\right\lrcorner \tau_{\mathrm{g}} .
$$

where as noted before, in this paper $\widetilde{\mathcal{A}}_{k}$ denotes the reverse of $\mathcal{A}_{k}$. Eq.(49) permits calculation of Hodge duals very easily in an orthonormal basis for which $\tau_{\mathrm{g}}=\boldsymbol{\theta}^{\mathbf{5}}$. Let $\left\{\vartheta^{\alpha}\right\}$ be the dual basis of $\left\{e_{\alpha}\right\}$ (i.e., it is a basis for $T^{*} U \equiv \bigwedge^{1} T^{*} U$ ) which is either orthonormal or a coordinate basis. Then writing $\mathrm{g}\left(\vartheta^{\alpha}, \vartheta^{\beta}\right)=g^{\alpha \beta}$, with $g^{\alpha \beta} g_{\alpha \rho}=\delta_{\rho}^{\beta}$, and $\vartheta^{\mu_{1} \ldots \mu_{p}}=\vartheta^{\mu_{1}} \wedge \ldots \wedge \vartheta^{\mu_{p}}, \vartheta^{\nu_{p+1} \ldots \nu_{n}}=\vartheta^{\nu_{p+1}} \wedge \ldots \wedge \vartheta^{\nu_{n}}$ we have from Eq.(49)

$$
\star \vartheta^{\mu_{1} \ldots \mu_{p}}=\frac{1}{(n-p) !} \sqrt{|\mathbf{g}|} g^{\mu_{1} \nu_{1}} \ldots g^{\mu_{p} \nu_{p}} \epsilon_{\nu_{1} \ldots \nu_{n}} \vartheta^{\nu_{p+1} \ldots \nu_{n}} .
$$

where $\mathbf{g}$ denotes the determinant of the matrix with entries $g_{\alpha \beta}=\mathbf{g}\left(e_{\alpha}, e_{\beta}\right)$, i.e., $\mathbf{g}=\operatorname{det}\left[g_{\alpha \beta}\right]$. We also define the inverse $\star^{-1}$ of the Hodge dual operator, such that $\star^{-1} \star=\star \star^{-1}=1$. It is given by:

$$
\begin{aligned}
& \star^{-1}: \sec \bigwedge^{n-r} T^{*} M \rightarrow \sec \bigwedge^{r} T^{*} M, \\
& \star^{-1}=(-1)^{r(n-r)} \operatorname{sgn} \mathbf{g} \star
\end{aligned}
$$

where sgn $\mathbf{g}=\mathbf{g} /|\mathbf{g}|$ denotes the sign of the determinant $\mathbf{g}$.

Some useful identities (used in the text) involving the Hodge star operator, the exterior product and contractions are:

$$
\begin{aligned}
& A_{r} \wedge \star B_{s}=B_{s} \wedge \star A_{r} ; \quad r=s \\
& A_{r} \cdot \star B_{s}=B_{s} \cdot \star A_{r} ; \quad r+s=n \\
& \left.A_{r} \wedge \star B_{s}=(-1)^{r(s-1)} \star\left(\tilde{A}_{r}\right\lrcorner B_{s}\right) ; \quad r \leq s \\
& \left.A_{r}\right\lrcorner \star B_{s}=(-1)^{r s} \star\left(\tilde{A}_{r} \wedge B_{s}\right) ; \quad r+s \leq n \\
& \star \tau_{g}=\operatorname{sign} \mathbf{g} ; \quad \star 1=\tau_{g} .
\end{aligned}
$$

\section{A.1.2 Dirac Operator Associated to a Levi-Civita Connection}

Let $d$ and $\delta$ be respectively the differential and Hodge codifferential operators acting on sections of $\mathcal{C}(M, \mathrm{~g})$. If $A_{p} \in \sec \bigwedge^{p} T^{*} M \hookrightarrow \sec \mathcal{C}(M, \mathrm{~g})$, then $\delta A_{p}=$ $(-1)^{p} \star^{-1} d \star A_{p}$

The Dirac operator acting on sections of $\mathcal{C}(M, \mathrm{~g})$ associated with the metric compatible connection $D$ is the invariant first order differential operator

$$
\boldsymbol{\partial}=\vartheta^{\alpha} D_{e_{\alpha}},
$$


where $\left\{e_{\alpha}\right\}$ is an arbitrary (coordinate or orthonormal) basis for $T U \subset T M$ and $\left\{\vartheta^{\alpha}\right\}$ is a basis for $T^{*} U \subset T^{*} M$ dual to the basis $\left\{e_{\alpha}\right\}$, i.e., $\vartheta^{\beta}\left(e_{\alpha}\right)=\delta_{\beta}^{\alpha}$, $\alpha, \beta=0,1,2,3$. The reciprocal basis of $\left\{\vartheta^{\alpha}\right\}$ is denoted $\left\{\vartheta_{\alpha}\right\}$ and we have $\vartheta_{\alpha} \cdot \vartheta_{\beta}=g_{\alpha \beta}$. Also,

$$
D_{e_{\alpha}} \vartheta^{\beta}=-L_{\alpha \lambda}^{\beta} \vartheta^{\lambda}
$$

and we define the connection 1-forms in the gauge defined by $\left\{\vartheta^{\alpha}\right\}$ as

$$
L_{\beta}^{\alpha}:=L_{\lambda \beta}^{\alpha} \vartheta^{\lambda} .
$$

We recall also that for an orthonormal basis it is usual to write $(\mathbf{a}, \mathbf{b}, \mathbf{c}=$ $0,1,2,3)$

$$
D_{\mathbf{e}_{\mathbf{a}}} \boldsymbol{\theta}^{\mathbf{b}}=-\omega_{\mathbf{a c}}^{\mathbf{b}} \theta^{\mathbf{c}}, \omega_{\mathbf{b}}^{\mathbf{a}}:=\omega_{\mathbf{c b}}^{\mathbf{a}} \theta^{\mathbf{c}} .
$$

Moreover, we write for an arbitrary tensor field $Y=Y_{\nu_{1} \ldots \nu_{s}}^{\mu_{1} \ldots \mu_{r}} \gamma^{\nu_{1}} \otimes \ldots \otimes \gamma^{\nu_{s}} \otimes$ $\partial_{\mu_{1}} \otimes \ldots \otimes \partial_{\mu_{r}}$ in a coordinate basis (and use the notation of Section 2 for the basis of the tangent and cotangent bundles),

$$
D_{\mathbf{e}_{\alpha}} Y:=\left(D_{\alpha} Y_{\nu_{1} \ldots \nu_{s}}^{\mu_{1} \ldots \mu_{r}}\right) \boldsymbol{\gamma}^{\nu_{1}} \otimes \ldots \otimes \boldsymbol{\gamma}^{\nu_{s}} \otimes \partial_{\mu_{1}} \otimes \ldots \otimes \partial_{\mu_{r}}
$$

We have also the important results (see, e.g., 9]) for the Dirac operator associated with the Levi-Civita connection $D$ acting on the sections of the Clifford bundle

$$
\begin{aligned}
\boldsymbol{\partial} A_{p} & \left.=\boldsymbol{\partial} \wedge A_{p}+\boldsymbol{\partial}\right\lrcorner A_{p}=d A_{p}-\delta A_{p}, \\
\boldsymbol{\partial} \wedge A_{p} & \left.=d A_{p}, \quad \boldsymbol{\partial}\right\lrcorner A_{p}=-\delta A_{p} .
\end{aligned}
$$

We shall need the following identity valid for any $A, B \in \sec \bigwedge^{1} T^{*} M \hookrightarrow$ $\mathcal{C} \ell(M, \mathrm{~g})$,

$$
\boldsymbol{\partial}(A \cdot B)=(A \cdot \boldsymbol{\partial}) B+(B \cdot \boldsymbol{\partial}) A+A\lrcorner(\boldsymbol{\partial} \wedge B)+B\lrcorner(\boldsymbol{\partial} \wedge A) .
$$

\section{A.2 Covariant D' Alembertian, Hodge D'Alembertian and Ricci Operators}

The square of the Dirac operator $\diamond=\partial^{2}$ is called Hodge D'Alembertian and we have the following noticeable formulas:

$$
\partial^{2}=-d \delta-\delta d
$$

and

$$
\boldsymbol{\partial}^{2} A_{p}=\boldsymbol{\partial} \cdot \boldsymbol{\partial} A_{p}+\boldsymbol{\partial} \wedge \boldsymbol{\partial} A_{p}
$$

where $\boldsymbol{\partial} \cdot \boldsymbol{\partial}$ is called the covariant D'Alembertian and $\boldsymbol{\partial} \wedge \boldsymbol{\partial}$ is called the Ricci operator If $A_{p}=\frac{1}{p !} A_{\mu_{1} \ldots \mu_{p}} \gamma^{\mu_{1}} \wedge \ldots \wedge \gamma^{\mu_{p}}$, we have

$$
\boldsymbol{\partial} \cdot \boldsymbol{\partial} A_{p}=g^{\alpha \beta}\left(D_{\partial_{\alpha}} D_{\partial_{\beta}}-\Gamma_{\alpha \beta}^{\rho} D_{\partial_{\rho}}\right) A_{p}=\frac{1}{p !} g^{\alpha \beta} D_{\alpha} D_{\beta} A_{\alpha_{1} \ldots \alpha_{p}} \gamma^{\alpha_{1}} \wedge \ldots \wedge \gamma^{\alpha_{p}}
$$

\footnotetext{
${ }^{4}$ For more details concerning the square of Dirac (and spin-Dirac operators) on a general Riemann-Cartan spacetime, see [10]
} 
Also for $\boldsymbol{\partial} \wedge \boldsymbol{\partial}$ in an arbitrary basis (coordinate or orthonormal)

$$
\boldsymbol{\partial} \wedge \boldsymbol{\partial} A_{p}=\frac{1}{2} \vartheta^{\alpha} \wedge \vartheta^{\beta}\left(\left[D_{e_{\alpha}}, D_{e_{\beta}}\right]-\left(L_{\alpha \beta}^{\rho}-L_{\beta \alpha}^{\rho}\right) D_{e_{\rho}}\right) A_{p} .
$$

In particular we show [9]) now that

$$
\partial \wedge \boldsymbol{\partial} \vartheta^{\mu}=\mathcal{R}^{\mu}
$$

where $\mathcal{R}^{\mu}=R_{\nu}^{\mu} \vartheta^{\nu} \in \sec \bigwedge^{1} T^{*} M \hookrightarrow \sec \mathcal{C}(M, \mathrm{~g})$ are the Ricci 1-form fields, such that if $R_{\nu}^{\mu}{ }_{\sigma \mu}$ are the components of the Riemann tensor we use the convention that $R_{\nu \sigma}=R_{\nu \sigma \mu}^{\mu}$ are the components of the Ricci tensor.

Applying this operator to the 1 -forms of the a 1 -form of the basis $\left\{\vartheta^{\mu}\right\}$, we get:

$$
(\phi \wedge \phi) \vartheta^{\mu}=-\frac{1}{2} R_{\rho}{ }^{\mu}{ }_{\alpha \beta}\left(\vartheta^{\alpha} \wedge \vartheta^{\beta}\right) \vartheta^{\rho}=-\mathcal{R}_{\rho}^{\mu} \vartheta_{\rho},
$$

Then,

$$
\mathcal{R}_{\rho}^{\mu} \vartheta^{\rho}=\mathcal{R}_{\rho}^{\mu}\left\llcorner\boldsymbol{\theta}^{\rho}+\mathcal{R}_{\rho}^{\mu} \wedge \boldsymbol{\theta}^{\rho} .\right.
$$

The second term in the r.h.s. of this equation is identically null due the first Bianchi identity. Then

$$
\begin{aligned}
\mathcal{R}_{\rho}^{\mu}\left\llcorner\vartheta^{\rho}\right. & \left.\left.=\vartheta^{\rho}\right\lrcorner \mathcal{R}_{\rho}^{\mu}=-\frac{1}{2} \vartheta^{\rho}\right\lrcorner\left(R_{\rho}{ }^{\mu \alpha}{ }_{\alpha \beta} \vartheta \wedge \vartheta^{\beta}\right) \\
& =\frac{1}{2} R_{\rho}{ }^{\mu}{ }_{\alpha \beta}\left(g^{\rho \alpha} \vartheta^{\beta}-g^{\rho \beta} \vartheta^{\alpha}\right) \\
& =g^{\rho \alpha} R_{\rho}{ }^{\mu}{ }_{\alpha \beta} \vartheta^{\beta}=R_{\beta}^{\mu} \vartheta^{\beta}=\mathcal{R}^{\mu},
\end{aligned}
$$

and Eq.(64) is proved.

We next show that for every $A \in \sec \bigwedge^{1} T^{*} M \hookrightarrow \sec \mathcal{C}(M, \mathrm{~g})$

$$
\phi \wedge \phi A=A_{\mu} \phi \wedge \phi \vartheta^{\mu} \text {. }
$$

Indeed, using Eq. (63) we can write (using for simplicity a coordinate basis) :

$$
\begin{aligned}
& \phi \wedge \phi A \\
& =\frac{1}{2} \gamma^{\alpha} \wedge \gamma^{\beta}\left\{\left[\partial_{\alpha}, \partial_{\beta}\right]\left(A_{\kappa}\right)-\Gamma_{\alpha \beta}^{\rho} \partial_{\rho}\left(A_{\kappa}\right)+\Gamma_{\beta \alpha}^{\rho} \partial_{\rho}\left(A_{\kappa}\right)\right\} \gamma^{\kappa}+A_{\mu} \phi \wedge \phi \gamma^{\mu} \\
& =A_{\mu} \phi \wedge \phi \gamma^{\mu} .
\end{aligned}
$$

\section{References}

[1] Benn, I. M., Conservation Laws in Arbitrary Space-times, Ann. Inst. H. Poincaré A XXXVII, 67-91 (1982).

[2] Eddington, A .S., The Mathematical Theory of Relativity (third edition), Chelsea Publ. Co, New York, 1975. 
[3] Fayo, F., and Sopuerta, C. F., On the Papapetrou Field in Vacuum, Class. Quant. Grav. 16, 2965-2985 (1999)

[4] Komar, A., Asymptotic Covariant Laws for Gravitational Radiation, Phys. Rev. 127, 1411-1418 (1962).

[5] Lawson, H. Blaine, Jr. and Michelson, M. L., Spin Geometry, Princeton University Press, Princeton, 1989.

[6] Mosna, R. A. and Rodrigues, W. A., Jr., The Bundles of Algebraic and DiracHestenes Spinor Fields, J. Math. Phys 45, 2945-2966 (2004). math-ph/0212033

[7] Notte-Cuello, E. A., Rodrigues, W. A. Jr., and da Rocha, R., The Effective Lorentzian and Teleparallel Spacetimes Generated by a Free Electromagnetic Field, in publ. Rep. Math. Phys. (2008). gr-qc/0612098

[8] Papapetrou, A., Champs Gravitationnels Stationaries à Symmetrie Axiale, Ann. Inst. H. Poincaré A IV, 83-105 (1966).

[9] Rodrigues, W. A. Jr. and Oliveira, E. Capelas, The Many Faces of Maxwell, Dirac and Einstein Equations. A Clifford Bundle Approach, Lecture Notes in Physics 722, Springer, Heidelberg 2007. http://www.ime.unicamp.br/ walrod/errata050408.pdf]

[10] Notte-Cuello, E. A., Rodrigues, W. A. Jr., and de Souza, Q. A. G., The Square of the Dirac and spin-Dirac Operators on a Riemann-Cartan Space(time), Rep. Math. Phys. 60, 135-157 (2007) math-ph/0703052

[11] Sachs, R. K., and Wu, H., General Relativity for Mathematicians, SpringerVerlag, New York 1977.

[12] Thirring, W., A Course in Mathematical Physics, volume 2., Springer-Verlag, New York, 1978. 